\title{
Der Faktor Q
}

Die Analphabeten des einundzwanzigsten Jahrhunderts werden nach Alvin Toffler diejenigen sein, die nicht lernen, verlernen und umlernen können. In kleinen und mittleren Unternehmen ist jedoch gerade der Faktor Qualifikation bzw. Lernen häufig eine zentrale Schwachstelle. Ausgehend von Projekterfahrungen werden Strategien zu ihrer Behebung vorgestellt.

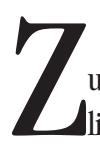

Von Petra Heuer und Holger Rohn ukunftsfähigkeit bedeutet ganz grundsätzlich, die Bedürfnisse heutiger Generationen zu befriedigen, ohne die Lebensgrundlagen kommender Generationen zu gefährden. Für Unternehmen heißt dies mit anderen Worten, die Wettbewerbsfähigkeit zu verbessern, Ressourcen zu schonen sowie soziale und ethische Verantwortung für Beschäftigte, Konsumenten und Nachbarn zu übernehmen - also eine dreifache Gewinnstrategie. Zukunftsfähiges Wirtschaften wird zunehmend von Seiten der Politik gefordert, eine breite Umsetzung in der Gesellschaft fehlt jedoch bislang.

Mitentscheidende Schwachstellen - insbesondere in kleinen und mittleren Unternehmen - sind nach wie vor oft die unzureichenden Qualifikationen der Beschäftigten und die mangelnde Einbindung ihres Know-hows in den betrieblichen Alltag. Für eine erfolgreiche Organisationsentwicklung und Sicherung der Wettbewerbsfähigkeit ist die Entwicklung der Humanressourcen eine ernst zu nehmende und zukunftsweisende Strategie. Dies zeigt auch die Evaluation zur Wirkung der hierauf zielenden Förderprogramme QUATRO und ADAPT (1): Unternehmen, die unabhängig von staatlichen Anreizsystemen in die Qualifikation der Beschäftigten investieren, waren hinsichtlich ihres wirtschaftlichen Erfolgs in jeder Beziehung am erfolgreichsten. Der wirtschaftliche Erfolg korreliert mit dem Investitionsverhalten bezüglich Personalentwicklung.

\section{- Trends der betrieblichen Weiterbildung}

Folgende Entwicklungen der betrieblichen Weiterbildung zeichnen sich ab (2):

- Die Bedeutung der Weiterbildung wird tendenziell weiter steigen. Dabei wird sich das absehbare Wachstum voraussichtlich bei den betriebsinternen Maßnahmen niederschlagen. Der Arbeitsplatz als Lernort erlebt eine deutliche Aufwertung.
Selbstgesteuertes Lernen mit Hilfe elektronischer Medien gewinnt weiter an Bedeutung. Schon jetzt steht Selbstlernen bei Fach- und Führungskräften an erster Stelle der Qualifizierungsmaßnahmen. Verlierer ist diesbezüglich die Beschäftigtengruppe der gering Qualifizierten, die diese Form der Weiterbildung häufig nicht nutzen können.

- Die Inhalte der beruflichen Weiterbildung verändern sich weg von Technik- und Fachwissen hin zu den sogenannten Schlüsselqualifikationen, da die „Halbwertszeit“ von Wissen sinkt. Teilnehmende sind dadurch weniger passiv Zuhörende, sondern Subjekte und damit aktiv Gestaltende ihres individuellen Lernprozesses.

- Weiterbildungsmaßnahmen werden immer häufiger genau auf den Bedarf zugeschnitten sein. Es wird eine Entwicklung von der Angebotsorientierung zur Nachfrageorientierung geben, sodass Weiterbildungsmaßnahmen eine größere Passgenauigkeit haben.

- Es zeichnet sich der Bedarf nach langfristiger angelegten und umfassenden Personalmanagementkonzepten $\mathrm{ab}$.

\section{Umweltmanagement qualifizieren}

Das Thema Umwelt gilt als ein wichtiger - wenn nicht sogar der wichtigste - Wegbereiter des Leitbildes Zukunftsfähige Entwicklung. Insbesondere im Bereich der Bildung hat über die Umweltbildung eine Entwicklung zur Bildung für Nachhaltigkeit stattgefunden (3). Hat die Einfuihrung von Umweltmanagementsystemen (UMS) in den vergangenen Jahren also zu einem maßgeblichen Bedeutungsgewinn der betrieblichen Weiterbildung beigetragen? Im Rahmen von UMS kommt der systematischen Qualifizierung und Einbindung der Beschäftigten eine zentrale Bedeutung zu (4). Trotzdem kann die Frage leider nicht mit einem deutlichen , ja“ beantwortet werden. Denn obwohl die Erfordernisse der Qualifizierung sowohl aus der EG-Audit-Verordnung als auch aus der ISO 14.000 deutlich hervor gehen, liegen in diesem Bereich selbst bei validierten bzw. zertifizierten Betrieben immer noch erhebliche Defizite (5). Dies zeigen auch Erfahrungen aus einem ADAPT-Projekt, das durch das Wuppertal-Institut betreut wurde (6).

\section{- Erfahrungen mit dem Faktor $Q$ im Umweltmanagement}

Die Unternehmen, die am Projekt teilgenommen haben, können als fortschrittlich bezeichnet werden, denn sie gehörten zu den ersten validierten KMU. Trotzdem ist auch bei ihnen die Qualifizierung der Beschäftigten ein vernachlässigter Faktor - sie erfolgt überwiegend unsystematisch und sporadisch. Dies betrifft alle Phasen der Qualifizierung, die Planung, Durchführung und Nachbereitung. Ein Budget für die Weiterbildung der Beschäftigten ist nur in seltenen Fällen fest eingeplant. Instrumente einer systematischen Analyse sind in den wenigsten Unternehmen vorhanden und die Wünsche der Mitarbeitenden werden nur selten und punktuell berücksichtigt. In vielen Unternehmen konzentriert sich die Qualifizierung - abgesehen von Unterweisungen am Arbeitsplatz überwiegend auf die Leitungsebene. Die Evaluation durchgeführter Qualifizierungsmaßnahmen findet in der Regel nicht statt, Erfahrungsberichte werden nicht veranlasst.

Inhaltlich dominieren technische und arbeitsaufgabenbezogene Qualifizierungen prägnant gegenüber sozialen und umweltbezogenen Qualifizierungen. Neben weit verbreiteten Mängeln in den Bereichen Basiswissen zum Umweltmanagement und innerbetriebliche Kommunikation, wurden ebenso Defizite in den gesetzlich geforderten Qualifizierungen, zum Beispiel der Gefahrstoffund Hygieneschulung, festgestellt. Neue Beschäftigte werden in den wenigsten Fällen systematisch über ihren engeren Arbeitsbereich hinaus informiert. So überrascht es nicht sonderlich, dass viele Beschäftigte äußern, über den betrieblichen Umweltschutz aber auch über andere Maßnahmen im Betrieb zu wenig informiert zu werden. Neben den Inhalten sind auch die Methoden, mit denen die Unternehmen arbeiten, sehr klassisch. Es dominieren nach wie vor Qualifizierungsformen, die auf einer reinen Informationsvermittlung beruhen, gegenüber aktiven Formen, wie etwa moderierten Arbeitsgruppen. Das Paradebeispiel ist die Unterweisung am Arbeitsplatz zu technischen Aspekten. Diese Formen aber werden von den Beschäftigten häufig als nicht ausreichend empfunden. Bei der Entwicklung und Durchführung bedarfsgerechter Qualifizierungen, die an 
die spezifischen Gegebenheiten im Unternehmen angepasst sind sowie neuere Methoden und umfangreichere Inhalte berücksichtigen, benötigen KMU deshalb Unterstïtzung.

\section{Ursachen der Schwachstellen}

Seitens der Unternehmen wird der betrieblichen Weiterbildung nach wie vor eine relativ geringe Bedeutung beigemessen. „Der Betrieb muss laufen“ lautet häufig das Motto. Dies impliziert jedoch vor allem eine kurzfristige Perspektive und bewirkt, dass die Unternehmen zumeist auf Anforderungen reagieren müssen, anstatt ihren Handlungsspielraum selbst aktiv zu gestalten. In den Unternehmen erfolgt also vor allem eine Reaktion auf Defizite, nur selten eine Ausrichtung auf zukünftige Bedarfe.

Eine Ursache ist darin zu sehen, dass KMU selten über ausreichende personelle, finanzielle und zeitliche Ressourcen verfügen, um Weiterbildung systematisch anzubieten oder durchzuführen. Da pädagogisches Know-how in KMU meist fehlt, muss es von externer Seite hinzugezogen werden. Auch die Zuständigkeit für die betriebliche Weiterbildung ist häufig nicht klar festgelegt.

Andererseits besteht jedoch auch in methodischer Hinsicht ein Defizit. Es mangelt an praktikablen Methoden, um den Qualifizierungsbedarf zu erheben. Der Transfer bestehender Ansätze, die in den letzten Jahren speziell für kleinere Unternehmen entwickelt wurden, wurde noch nicht in ausreichendem Maße geschaffen.

Darüber hinaus besteht auch ein angebotsseitiger Mangel - die angebotenen Qualifizierungen sind zwar vielfältig, häufig aber nicht auf die speziellen Bedarfe der Unternehmen zugeschnitten. Das bedeutet, dass für Unternehmen Schwierigkeiten darin bestehen, sich einerseits einen Überblick über das bestehende Angebot zu verschaffen und eine sichere Auswahl zu treffen sowie andererseits Maßnahmen zu finden, die gezielt auf die eigenen Bedürfnisse ausgerichtet sind.

\section{Einen Überblick über alle bisher erschienen Hefte des Informations- dienstes "Ökologisches Wirtschaften" finden Sie auf unseren Internetseiten unter der Adresse: www.oekom.de} Wir freuen uns auf Ihren Besuch!

\section{Faktor Q - ein Ausblick}

Um die beschriebenen Schwierigkeiten anzugehen, sind auf verschiedenen Ebenen Handlungsbedarfe festzustellen.

In der breiten Masse der Unternehmen fehlt die Einsicht, welche Bedeutung der Qualifizierung ihrer Beschäftigten gerade in einem Hochlohnland wie Deutschland zukommt, um die wirtschaftliche Situation ihres Unternehmens zu sichern. Einzelne Unternehmen haben diese Situation bereits erkannt und handeln dementsprechend. Eine neuere Überlegung besteht darin, dass mehrere KMU sich einen Personalentwickler teilen. So können die Kosten minimiert werden, während zugleich eine kontinuierliche Analyse des Qualifizierungsbedarfs und eine kompetente Beratung gewährleistet ist, die das fehlende Know-how sinnvoll ergänzt. Für die Beschäftigten selbst bedeutet es lernen zu müssen, ihre Qualifizierungswünsche zu artikulieren - insbesondere in den ausführenden Ebenen. Durch die Unternehmensführung können sie hierbei unterstützt werden.

Die Weiterbildungsträger sollten sich in Zukunft noch stärker darauf einstellen, Unternehmen „dort abzuholen, wo sie stehen“. Dies beinhaltet neben einer stärkeren Nachfrageorientierung zusätzlich ein größeres Maß an Beratungsdienstleistung und Lernprozessbegleitung (7). Veränderungen werden auch in Richtung eines Angebotes gefordert, das stärker in Moderations- und Mediationstechniken, in Teamfähigkeit und in Organisationsentwicklungsfragen qualifiziert (8).

In verschiedenen Städten haben die Bildungsträger bereits angefangen sich zu vernetzen, das Angebot in seinem Spektrum und seiner Qualität gebündelt zu präsentieren und zum Teil auch Beratungsdienstleistungen zu integrieren. Für Unternehmen ist eine solche Weiterbildungsberatung von neutraler Stelle hilfreich. Diese bestehenden Ansätze regionaler Netzwerke sollten ausgedehnt und verstetigt werden.

Von wissenschaftlicher Seite aus kann dieser Prozess zum einen unterstïtzt werden, indem die bestehenden Methoden der Qualifizierungsbedarfsanalysen für KMU in der Öffentlichkeit stärker publik gemacht werden. Zum anderen sollten die Methoden weiterentwickelt werden, sodass sie leichter zu handhaben sind. Sie bedürfen außerdem einer Ausgestaltung, die alle Aspekte einer zukunftsfähigen Entwicklung berïcksichtigt. Ein wichtiges Ziel sollte darin bestehen, die Qualifizierungsbedarfe von Unternehmen und Regionen vorausschauend zu ermitteln. Erst durch das Zu- sammenspiel der verschiedenen Faktoren kann so eine zukunftsfähige Unternehmensentwicklung auf breiter Basis verwirklicht werden.

\section{Anmerkungen}

(1) Vgl. www.bn.shuttle.de/adapt und www.gib.nrw.de.

(2) Vgl. Weif, Reinhold: Betriebliche Weiterbildung: Zahlen - Fakten - Trends. In: Geißler, Karlheinz A./ Looss, Wolfgang (Hrsg.): Handbuch Personalentwicklung. 54. Erg.Lfg. Köln 1999, Kap. 3.16; Wuppertaler Kreis e.V. (Hrsg.): Erfolgsfaktor Weiterbildung. Wie mittelständische Unternehmen ihre Leistungsfähigkeit und Innovationskraft steigern. Köln 1999.

(3) Vgl. Beyer, Axel (Hrsg.): Nachhaltigkeit und Umweltbildung, Hamburg 1998; Haan, Gerhard de/ Kuckartz, Udo (Hrsg.): Umweltbildung und Umweltbewußtsein. Forschungsperspektiven im Kontext nachhaltiger Entwicklung, Opladen 1998.

(4) Vgl. u.a. Umweltbundesamt (Hrsg.): Umweltmanagement in der Praxis. Texte 52/98. Berlin 1998.

(5) Vgl. u.a. Dyllick, Thomas/ Hamschmidt, Jost: Wirksamkeit und Leistung von Umweltmanagementsystemen. Zürich 2000.

(6) Informationen zum Projekt „Lokal handeln - systemweit denken" im Internet unter www.wupperinst.org/adapt (7) Vgl. u.a. BLK - Bund-Länder-Kommission für Bildungsplanung und Forschungsförderung: Bildung für eine nachhaltige Entwicklung - Orientierungsrahmen. Heft 69, Bonn 1998,

S. 60; Wuppertaler Kreis a.a.0.; Institut der deutschen Wirtschaft (Hrsg.): Betriebliche Weiterbildung. Mehr Teilnehmer - größere Wirtschaftlichkeit, Köln 1997; Weiß a.a.0.

(8) Haan, Gerhard de: Schlüsselkompetenz, Umweltsyndrome und Bildungsreform. In: Beyer, Axel/ Wass von Czege, Andreas (Hrsg.): Fähig für die Zukunft. Schlüsselqualifikationen für eine nachhaltige Entwicklung. Hamburg 1998.

\section{Die Autorlnnen}

Petra Hever ist wissenschaftliche Mitarbeiterin am Wuppertal Institut für Klima, Umwelt, Energie, AG Ökoeffizienz \& zukunftsfähige Unternehmen. Kontakt: Wuppertal Institut für Klima, Umwelt, Energie, Döppersberg 19, 42103 Wuppertal. Tel. 0202/ 2492-239, Fax -138, E-mail: petra.hever@wupperinst.org Holger Rohn ist Gesellschafter der Trifolium Beratungsgesellschaft für zukunftsfähiges Wirtschaften und freier Mitarbeiter am Wuppertal Institut für Klima, Umwelt, Energie.

Kontakt: Trifolium - Beratungsgesellschaft für zukunftsfähiges Wirtschaften, Alte Bahnhofstraße 13, 61169 Friedberg. Tel. 06031/ 143-36, Fax -10, E-mail: trifolium.rohn@t-online.de 
(c) 20I0 Authors; licensee IÖW and oekom verlag. This is an article distributed under the terms of the Creative Commons Attribution Non-Commercial No Derivates License (http://creativecommons.org/licenses/by-nc-nd/3.o/), which permits unrestricted use, distribution, and reproduction in any medium, provided the original work is properly cited. 